\title{
STABLE AND EFFICIENT SPECTRAL METHODS IN UNBOUNDED DOMAINS USING LAGUERRE FUNCTIONS*
}

\author{
JIE SHEN ${ }^{\dagger}$
}

\begin{abstract}
Stable and efficient spectral methods using Laguerre functions are proposed and analyzed for model elliptic equations on regular unbounded domains. It is shown that spectral-Galerkin approximations based on Laguerre functions are stable and convergent with spectral accuracy in the usual (not weighted) Sobolev spaces. Efficient, accurate, and well-conditioned algorithms using Laguerre functions are developed and implemented. Numerical results indicating the spectral convergence rate and effectiveness of these algorithms are presented.
\end{abstract}

Key words. Laguerre function, unbounded domain, spectral-Galerkin approximation, elliptic equation

AMS subject classifications. $65 \mathrm{~N} 35,65 \mathrm{~N} 22,65 \mathrm{~F} 05,35 \mathrm{~J} 05$

PII. S0036142999362936

1. Introduction. While the Legendre- or Chebyshev-spectral approximations for PDEs in bounded domains have achieved great success and popularity in recent years (see, e.g., $[10,5,2]$ ), spectral approximations for PDEs in unbounded domains have received only limited attention. Pioneer work on Laguerre approximation was developed in Gottlieb and Orszag [10] and Maday, Pernaud-Thomas, and Vandeven [15] (see also [9, 2]). Numerical investigations to model linear elliptic equations using Laguerre-collocation or Laguerre-tau approximations can be found in $[16,6,14]$. However, to the best of our knowledge, most of the previous numerical results used Laguerre polynomials which, from a theoretical point of view provide only meaningful results inside small intervals since the error estimate is obtained in weighted Sobolev spaces with an exponentially decaying weight, and from a practical point of view are not suitable for practical computations due to the extremely ill-conditioned behaviors of the Laguerre polynomials and of the Laguerre-Gauss-Radau quadrature formula. Furthermore, it was pointed out by Gottlieb and Orszag [10] that Laguerre polynomials/functions have very poor resolution properties when compared with other types of orthogonal polynomials. These are the three main factors which have limited the interests and developments on using Laguerre polynomials for problems in unbounded domains.

The aim of this paper is to develop effective remedies for the aforementioned difficulties associated with Laguerre approximations. More precisely, we will propose uniformly convergent Galerkin approximations using Laguerre functions for elliptic equations in regular unbounded domains, and construct stable and efficient numerical algorithms for their practical implementations.

Let us mention that in [13], a Galerkin method using Laguerre polynomials applied to a properly transformed equation is analyzed for some nonlinear PDEs. It turns out that the effect of transforming the equations as in [13] is equivalent to the Galerkin method using Laguerre functions presented in this paper. However, the analysis and

\footnotetext{
${ }^{*}$ Received by the editors October 8, 1999; accepted for publication (in revised form) May 28, 2000; published electronically September 20, 2000.

http://www.siam.org/journals/sinum/38-4/36293.html

${ }^{\dagger}$ Department of Mathematics, Penn State University, University Park, PA 16802 (shen@math.psu.edu). This work was partially supported by NFS grants DMS-9603200 and DMS9706951.
} 
implementation in [13] were based on cumbersome weighted variational forms, while the approach in this paper is based on a usual variational formulation, which is better suited for both the analysis and implementation and warrants a detailed study. We note also that recently Guo [12] has developed some interesting theoretical results on using Jacoby polynomials to approximate functions on the half line.

The paper is organized as follows. In the next section, we consider a weighted Galerkin method using Laguerre polynomials. We demonstrate by error estimates and by numerical examples that this method, due to the exponentially decaying weight in its variational formulation, provides only meaningful results within a small interval. Section 3 is the essential part of the paper. Here we investigate the Galerkin method using Laguerre functions based on the usual variational formulation. It is shown that this method provides a uniformly convergent result in the whole semi-infinite interval. Several important implementation aspects such as Galerkin method with numerical integration or collocation, preconditioning, and scaling are also addressed. In section 4, we briefly mention some immediate extensions to general boundary conditions and to multidimensional problems. Some concluding remarks are given in section 5. Finally, a stable discrete transform based on Laguerre functions is given in the appendix.

\section{Galerkin method using Laguerre polynomials.}

2.1. Basic properties of Laguerre polynomials. We recall that the Laguerre polynomials are defined by the three-term recurrence relation $L_{0}(x)=1, L_{1}(x)=1-x$, and

$$
L_{n}(x)=\frac{2 n-1-x}{n} L_{n-1}(x)-\frac{n-1}{n} L_{n-2}(x), \quad n \geq 2 .
$$

Some other useful relations are

$$
\begin{aligned}
& L_{n}(0)=1, L_{n}^{\prime}(0)=-k ; \\
& L_{n}^{\prime}(x)-L_{n+1}^{\prime}(x)=L_{n}(x), x L_{n+1}^{\prime}(x)=(n+1)\left(L_{n+1}(x)-L_{n}(x)\right), \\
& \quad \int_{0}^{\infty} L_{i}(x) L_{j}(x) e^{-x} d x=\delta_{i j} \quad \forall i, j \geq 0 .
\end{aligned}
$$

Let us now define some suitable functional spaces for Laguerre approximation. Let $\omega(x)=e^{-x}$ and $L_{\omega}^{2}\left(\mathcal{R}_{+}\right)=\left\{v:\|v\|_{L_{\omega}^{2}}<\infty\right\}$ which is a Hilbert space equipped with the following inner product and norm:

$$
(u, v)_{\omega}=\int_{0}^{\infty} u(x) v(x) \omega(x) d x, \quad\|u\|_{\omega}=(u, u)_{\omega}^{\frac{1}{2}} .
$$

For any nonnegative integer $m$, we define $H_{\omega}^{m}\left(\mathcal{R}_{+}\right)=\left\{v: \frac{d^{k} v}{d x^{k}} \in L_{\omega}^{2}\left(\mathcal{R}_{+}\right), 0 \leq\right.$ $k \leq m\}$, which are associated with the following seminorm and norm:

$$
|v|_{m, \omega}=\left\|\frac{d^{m} v}{d x^{m}}\right\|_{\omega}, \quad\|v\|_{m, \omega}=\left(\sum_{k=0}^{m}|v|_{k, \omega}^{2}\right)^{\frac{1}{2}} .
$$

We also denote in particular $H_{0, \omega}^{1}\left(\mathcal{R}_{+}\right)=\left\{v: v \in H_{\omega}^{1}\left(\mathcal{R}_{+}\right)\right.$and $\left.v(0)=0\right\}$.

For any $r>0$, the space $H_{\omega}^{r}\left(\mathcal{R}_{+}\right)$and its norm $\|v\|_{r, \omega}$ can be defined by interpolation as in Adams [1]. For $\beta \geq 0$, we also define $H_{\omega, \beta}^{r}\left(\mathcal{R}_{+}\right):=\left\{v \in H_{\omega}^{r}\left(\mathcal{R}_{+}\right): x^{\frac{\beta}{2}} v \in\right.$ 
$\left.H_{\omega}^{r}\left(\mathcal{R}_{+}\right)\right\}$which is equipped with the norm $\|v\|_{r, \omega, \beta}=\left\|v(1+x)^{\frac{\beta}{2}}\right\|_{r, \omega}$. As usual, $\omega$ will be omitted from the notations in the case of $\omega \equiv 1$. The following lemma is proved in [13].

LEMMA 2.1.

$$
\begin{aligned}
\left\|e^{-\frac{x}{2}} v\right\|_{L^{\infty}\left(\mathcal{R}_{+}\right)} & \leq \sqrt{2}\|v\|_{\omega}^{\frac{1}{2}}|v|_{1, \omega}^{\frac{1}{2}}, \quad \forall v \in H_{0, \omega}^{1}\left(\mathcal{R}_{+}\right) . \\
\|v\|_{\omega} & \leq 2|v|_{1, \omega},
\end{aligned}
$$

Let $N$ be any positive integer, and $P_{N}$ be the space of polynomials of degree at most $N$; we set $X_{N}=P_{N} \cap H_{0, \omega}^{1}\left(\mathcal{R}_{+}\right)$. Let $\pi_{N}: L_{\omega}^{2}\left(\mathcal{R}_{+}\right) \rightarrow P_{N}$ be the $L_{\omega}^{2}$-orthogonal projector, i.e., for any $v \in L_{\omega}^{2}\left(\mathcal{R}_{+}\right),\left(v-\pi_{N} v, \phi\right)_{\omega}=0 \forall \phi \in P_{N}$.

Hereafter, the expression " $A_{N} \lesssim B_{N}$ " means that there exists a constant $C$ independent of $N$ such that (s.t.) " $A_{N} \leq C B_{N}$."

LEMMA 2.2. Let $r \geq 0$ and $\beta$ is the largest integer for which $\beta<r+1$. Then

$$
\left\|v-\pi_{N} v\right\|_{\mu, \omega} \lesssim N^{\mu-\frac{r}{2}}\|v\|_{r, \omega, \beta} \quad \forall v \in H_{\omega, \beta}^{r}\left(\mathcal{R}_{+}\right) \text {and } 0 \leq \mu \leq r .
$$

A proof of the above lemma can be found in [2]. A slightly different form of this result was first presented in Maday, Pernaud-Thomas, and Vandeven [15] (see also $[9])$.

We now define the $H_{0, \omega}^{1}$-projector $\pi_{N}^{1,0}: H_{0, \omega}^{1}\left(\mathcal{R}_{+}\right) \rightarrow X_{N}$ by

$$
\left(\frac{d}{d x}\left(v-\pi_{N}^{1,0} v\right), \frac{d}{d x} \phi\right)_{\omega}=0 \quad \forall \phi \in X_{N}, v \in H_{0, \omega}^{1}\left(\mathcal{R}_{+}\right) .
$$

Lemma 2.3. Let $r \geq 1$ and $\beta$ be the largest integer for which $\beta<r$. Then

$$
\left\|v-\pi_{N}^{1,0} v\right\|_{1, \omega} \lesssim N^{\frac{1}{2}-\frac{r}{2}}\left\|v_{x}\right\|_{r-1, \omega, \beta} \forall v \text { s.t. } v \in H_{0, \omega}^{1}\left(\mathcal{R}_{+}\right), v_{x} \in H_{\omega, \beta}^{r-1}\left(\mathcal{R}_{+}\right) .
$$

Proof. This lemma is a slight improvement of a similar result in [13]. It follows with the same argument as in the proof of Lemma 2.4 in [13]. Indeed, given $v \in$ $H_{0, \omega}^{1}\left(\mathcal{R}_{+}\right)$, we set $v_{N}(x)=\int_{0}^{x} \pi_{N-1} v_{y} d y$. Then $v_{N} \in X_{N}$. Thanks to Lemmas 2.1 and 2.2 ,

$$
\left\|v-\pi_{N}^{1,0} v\right\|_{1, \omega} \lesssim\left|v-v_{N}\right|_{1, \omega}=\left\|v_{x}-P_{N-1} v_{x}\right\|_{\omega} \lesssim N^{\frac{1-r}{2}}\left\|v_{x}\right\|_{r-1, \omega, \beta} .
$$

2.2. A (weighted) Galerkin method. We consider the approximation of the following model equation:

$$
-\frac{d^{2} u}{d x^{2}}+\alpha u=f, x \in \mathcal{R}_{+} ; u(0)=0 .
$$

A weighted Laguerre-Galerkin approximation for (2.4) is the following: Find $u_{N} \in X_{N}$ such that

$$
\left(\frac{d u_{N}}{d x}, \frac{d v}{d x}\right)_{\omega}-\left(\frac{d u_{N}}{d x}, v\right)_{\omega}+\alpha\left(u_{N}, v\right)_{\omega}=(f, v)_{\omega} \forall v \in X_{N} .
$$

THEOREM 2.1. Let $u$ and $u_{N}$ be, respectively, the solutions of (2.4) and (2.5). Let $\alpha>\frac{1}{4}, r \geq 1$, and $\beta$ be the largest integer for which $\beta<r$. Then

$$
\left\|u-u_{N}\right\|_{1, \omega} \leq c N^{\frac{1}{2}-\frac{r}{2}}\left\|u_{x}\right\|_{r-1, \omega, \beta} .
$$



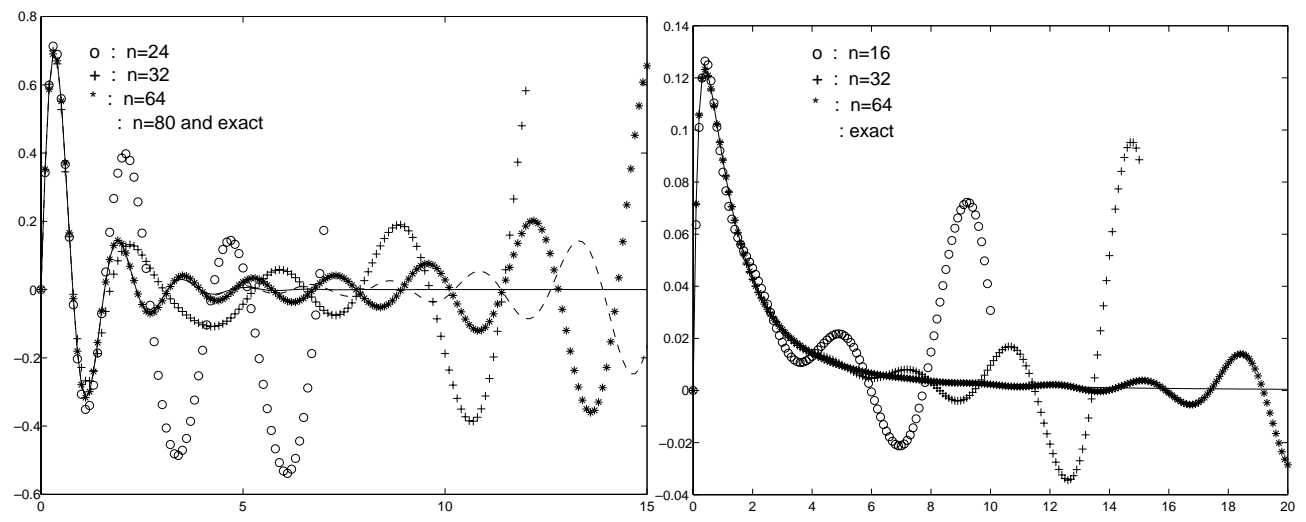

FIG. 2.1. Galerkin approximation using Laguerre polynomials: Example 1 with $k=4$ is on the left and Example 2 with $h=3.5$ is on the right.

Proof. Let $e_{N}=u_{N}-\pi_{N}^{1,0} u$. Subtracting (2.4) from (2.5), we find

$$
\begin{aligned}
\left(\frac{d e_{N}}{d x}, \frac{d v}{d x}\right)_{\omega} & -\left(\frac{d e_{N}}{d x}, v\right)_{\omega}+\alpha\left(e_{N}, v\right)_{\omega} \\
= & -\left(\frac{d}{d x}\left(u-\pi_{N}^{1,0} u\right), v\right)_{\omega}+\alpha\left(u-\pi_{N}^{1,0} u, v\right)_{\omega} \\
= & \left(\left(u-\pi_{N}^{1,0} u\right), \frac{d v}{d x}\right)_{\omega}+(\alpha-1)\left(u-\pi_{N}^{1,0} u, v\right)_{\omega} \quad \forall v \in X_{N} .
\end{aligned}
$$

Let us denote $\delta=\min \{1,4 \alpha-1\}$ which is positive if $\alpha>\frac{1}{4}$. Setting $v=e_{N}$ in the above equation and using Lemma 2.2 and the fact that $\left(\frac{d e_{N}}{d x}, e_{N}\right)_{\omega}=\frac{1}{2}\left(e_{N}, e_{N}\right)_{\omega}$, we find

$$
\delta\left|e_{N}\right|_{1, \omega}^{2} \leq\left|e_{N}\right|_{1, \omega}^{2}-\left(\frac{1}{2}-\alpha\right)\left\|e_{N}\right\|_{\omega}^{2} \leq|\alpha-1|\left\|u-\pi_{N}^{1,0} u\right\|_{\omega}\left\|e_{N}\right\|_{\omega},
$$

which implies that

$$
\sqrt{\delta}\left|e_{N}\right|_{1, \omega} \lesssim\left\|u-\pi_{N}^{1,0} u\right\|_{\omega} \lesssim\left\|u-\pi_{N}^{1,0} u\right\|_{1, \omega} .
$$

We then conclude by using the triangular inequality.

Remark 2.1. The above results are established without assuming any condition at infinity on the forcing function $f$ and the exact solution $u$. Consequently, these results do not provide any meaningful estimate for $x$ large. Indeed, the only estimate on point-wise error we can derive from Lemma 2.1 and Theorem 2.1 is that $\forall x \in \mathcal{R}_{+}$,

$$
\left|\left(u-u_{N}\right)(x)\right| \leq c e^{\frac{x}{2}} N^{\frac{1}{2}-\frac{r}{2}}\left\|u_{x}\right\|_{r-1, \omega, \beta} \quad \forall x \in \mathcal{R}_{+} .
$$

Thus, the point-wise error may grow exponentially with respect to $x$. On the other hand, this approximation using Laguerre polynomials is capable of providing spectrally convergent results within a fixed interval, regardless of the solution's behavior at infinity as long as its growth rate is bounded by $e^{c x}$ with some $c<\frac{1}{2}$. 
In order to examine numerically the convergence behavior, we consider the following two exact solutions of (2.4):

Example 1. $u(x)=\sin k x e^{-x}$ (exponential decay at infinity).

Example 2. $u(x)=\frac{x}{(1+x)^{h}}$ (algebraic decay without essential singularity at infinity).

In Figure 2.1, we compare the exact solutions with approximate solutions obtained by direct Laguerre-Galerkin method to (2.4) using different numbers of modes $n$. As expected, the direct Laguerre-Galerkin method leads only to meaningful approximation on a relatively short interval while the approximations for $x$ large diverge exponentially.

3. Galerkin methods using Laguerre functions. The problem with the above formulation is that the exponentially decaying weight associated with the variational formulation renders the approximate solution useless for large $x$. Thus the Laguerre polynomials are not suitable for practical numerical computations. Instead, the so-called Laguerre functions should be used.

3.1. Laguerre functions. We recall that the $n$th degree Laguerre function is defined as $\hat{L}_{n}(x)=L_{n}(x) e^{-x / 2}$ which forms a sequence of orthogonal basis in $L^{2}\left(R_{+}\right)$ since

$$
\int_{0}^{+\infty} \hat{L}_{n}(x) \hat{L}_{m}(x) d x=\delta_{n m} .
$$

We emphasize that in contrast to Laguerre polynomials, the Laguerre functions are well behaved, as indicated by the following relations (cf. page 40 in [7]):

$$
\left|\hat{L}_{n}(x)\right| \leq 1 \quad \forall x \in R_{+}, n=0,1,2, \ldots,
$$

and (cf. Thm. 8.22.1 in [22])

$$
\hat{L}_{n}(x)=\pi^{-1 / 2}(n x)^{-1 / 4} \cos \left\{2(n x)^{1 / 2}-\frac{\pi}{4}\right\}+O\left(e^{-x / 2} n^{-3 / 4}\right) .
$$

Lemma 3.1. For $r, \beta \geq 0$, the mapping $\phi \rightarrow \phi e^{-x / 2}$ is an isomorphism from $H_{\omega, \beta}^{r}\left(\mathcal{R}_{+}\right)$onto $H_{\beta}^{r}\left(\mathcal{R}_{+}\right)$. A proof of the lemma in the case of $\beta=0$ was given in [15] and [2]. The extension to $\beta>0$ is obvious.

We now define some approximation spaces and corresponding projection operators related to the series in Laguerre functions. We set

$$
\hat{P}_{N}=\left\{u: u=v e^{-x / 2} \text { with } v \in P_{N}\right\}, \hat{X}_{N}=\left\{u: u=v e^{-x / 2} \text { with } v \in X_{N}\right\},
$$

and we define $\hat{\pi}_{N}: L^{2}\left(\mathcal{R}_{+}\right) \rightarrow \hat{P}_{N}$ and $\hat{\pi}_{N}^{1,0}: H^{1}\left(\mathcal{R}_{+}\right) \rightarrow \hat{X}_{N}$, respectively, by

$$
\hat{\pi}_{N} u=e^{-x / 2} \pi_{N}\left(u e^{x / 2}\right), \hat{\pi}_{N}^{1,0} u=e^{-x / 2} \pi_{N}^{1,0}\left(u e^{x / 2}\right) .
$$

The following lemma characterizes $\hat{\pi}_{N}$ and $\hat{\pi}_{N}^{1,0}$.

LEMMA 3.2.

$$
\begin{aligned}
& \left(u-\hat{\pi}_{N} u, v_{N}\right)=0 \forall v_{N} \in \hat{P}_{N}, u \in L^{2}\left(R_{+}\right) \\
& \left(\frac{d}{d x}\left(u-\hat{\pi}_{N}^{1,0} u\right), \frac{d}{d x} v_{N}\right)+\frac{1}{4}\left(u-\hat{\pi}_{N}^{1,0} u, v_{N}\right)=0 \forall v_{N} \in \hat{X}_{N}, u \in H_{0}^{1}\left(R_{+}\right) .
\end{aligned}
$$


Proof. The first identity is obvious. To prove the second identity, we write

$$
\begin{aligned}
\frac{d}{d x}\left(u-\hat{\pi}_{N}^{1,0} u\right) & =\frac{d}{d x}\left[e^{-x / 2}\left(u e^{x / 2}\right)-e^{-x / 2} \pi_{N}^{1,0}\left(u e^{x / 2}\right)\right] \\
& =e^{-x / 2}\left\{\left[u e^{x / 2}-\pi_{N}^{1,0}\left(u e^{x / 2}\right)\right]^{\prime}-\frac{1}{2}\left[u e^{x / 2}-\pi_{N}^{1,0}\left(u e^{x / 2}\right)\right]\right\} .
\end{aligned}
$$

Setting $\omega=e^{-x}$, using the definition of $\pi_{N}^{1,0}$, and integration by parts, we find that for any $\hat{v}_{N}=v_{N} e^{-x / 2}$ with $v_{N} \in X_{N}$,

$$
\begin{aligned}
\left(\frac{d}{d x}\left(u-\hat{\pi}_{N}^{1,0} u\right), \hat{v}_{N}^{\prime}\right) & =\left(\left[u e^{x / 2}-\pi_{N}^{1,0}\left(u e^{x / 2}\right)\right]^{\prime}-\frac{1}{2}\left[u e^{x / 2}-\pi_{N}^{1,0}\left(u e^{x / 2}\right)\right], v_{N}^{\prime}-\frac{1}{2} v_{N}\right)_{\omega} \\
& =-\frac{1}{2} \int_{0}^{+\infty}\left[\left(u e^{x / 2}-\pi_{N}^{1,0}\left(u e^{x / 2}\right)\right) v_{N}\right]^{\prime} e^{-x} d x \\
& +\frac{1}{4}\left(\left[u e^{x / 2}-\pi_{N}^{1,0}\left(u e^{x / 2}\right)\right], v_{N}\right)_{\omega} \\
& =-\frac{1}{4}\left(\left[u e^{x / 2}-\pi_{N}^{1,0}\left(u e^{x / 2}\right)\right], v_{N}\right)_{\omega}=-\frac{1}{4}\left(u-\hat{\pi}_{N}^{1,0} u, \hat{v}_{N}\right),
\end{aligned}
$$

which implies the second identity.

LEMMA 3.3.

$$
\left\|u-\hat{\pi}_{N} u\right\|_{\mu} \lesssim N^{\mu-\frac{r}{2}}\|u\|_{r, \beta} \forall u \in H_{\beta}^{r}\left(\mathcal{R}_{+}\right) \quad \text { and } 0 \leq \mu \leq r,
$$

where $r \geq 0, \beta$ is the largest integer for which $\beta<r+1$; and

$$
\left\|u-\hat{\pi}_{N}^{1,0} u\right\|_{1} \lesssim N^{\frac{1-r}{2}}\left\|u_{x}+\frac{1}{2} u\right\|_{r-1, \beta} \quad \forall u \text { s.t. } u_{x}+\frac{1}{2} u \in H_{\beta}^{r-1}\left(\mathcal{R}_{+}\right), u \in H_{0}^{1}\left(R_{+}\right),
$$

where $r \geq 1, \beta$ is the largest integer for which $\beta<r$.

Proof. The above results are direct consequences of Lemmas 2.2, 2.3, and 3.1. In fact,

$$
\begin{aligned}
\left\|u-\hat{\pi}_{N} u\right\|_{\mu} & =\left\|\left[u e^{x / 2}-\pi_{N}\left(u e^{x / 2}\right)\right] e^{-x / 2}\right\|_{\mu}=\left\|\left[u e^{x / 2}-\pi_{N}\left(u e^{x / 2}\right)\right]\right\|_{\mu, \omega} \\
& \lesssim N^{\mu-r / 2}\left\|u e^{x / 2}\right\|_{r, \beta, \omega} \lesssim N^{\mu-r / 2}\|u\|_{r, \beta} .
\end{aligned}
$$
$\left.\frac{1}{2} u\right)$.

The second inequality can be proved in a similar way since $\left(u e^{x / 2}\right)_{x}=e^{x / 2}\left(u_{x}+\right.$

3.2. Galerkin method. Since we will be working with the usual Sobolev spaces, it is necessary to require that the solution tends to zero at infinity. Hence, instead of (2.4), we consider the following problem:

$$
-\frac{d^{2} u}{d x^{2}}+\alpha u=f, x \in \mathcal{R}_{+} ; u(0)=0, \lim _{x \rightarrow+\infty} u(x)=0 .
$$

We consider the following Galerkin approximation to (3.7):

Find $u_{N} \in \hat{X}_{N}$ such that

$$
\left(u_{N}^{\prime}, v^{\prime}\right)+\alpha\left(u_{N}, v\right)=(f, v) \forall v \in \hat{X}_{N} .
$$


It is clear that for $\alpha>0$ the problems (3.7) and (3.8) admit an unique solution since $a(u, u)=\left|u_{x}\right|_{1}^{2}+\alpha\|u\|^{2} \geq \min (1, \alpha)\|u\|_{1}^{2} \forall u \in H_{0}^{1}\left(R_{+}\right)$.

Theorem 3.1. Let $\alpha>0, r \geq 1$, and $\beta$ be the largest integer for which $\beta<r$. Then

$$
\left\|u-u_{N}\right\|_{1} \lesssim N^{\frac{1-r}{2}}\left\|u_{x}+\frac{1}{2} u\right\|_{r-1, \beta}
$$

Proof. We derive from (3.7) and Lemma 3.2 that

$$
\left(\left(\hat{\pi}_{N}^{1,0} u\right)^{\prime}, v^{\prime}\right)+\alpha\left(\hat{\pi}_{N}^{1,0} u, v\right)=-\left(\alpha-\frac{1}{4}\right)\left(u-\hat{\pi}_{N}^{1,0} u, v\right)+(f, v) \forall v \in \hat{X}_{N} .
$$

Let $e_{N}=u_{N}-\hat{\pi}_{N}^{1,0} u$. Subtracting the above from (3.8), we find

$$
\left(e_{N}^{\prime}, v^{\prime}\right)+\alpha\left(e_{N}, v\right)=\left(\alpha-\frac{1}{4}\right)\left(u-\hat{\pi}_{N}^{1,0} u, v\right) \forall v \in \hat{X}_{N} .
$$

Taking $v=e_{N}$ in the above relation, we derive by Schwartz inequality that

$$
\left|e_{N}^{\prime}\right|_{1}^{2}+\alpha\left\|e_{N}\right\|^{2}=\left(\alpha-\frac{1}{4}\right)\left(u-\hat{\pi}_{N}^{1,0} u, e_{N}\right) \leq \frac{\alpha}{2}\left\|e_{N}\right\|^{2}+\frac{\left(\alpha-\frac{1}{4}\right)^{2}}{2 \alpha}\left\|u-\hat{\pi}_{N}^{1,0} u\right\|^{2} .
$$

The desired result follows immediately from Lemma 3.3 and the triangular inequality.

As for the implementation, we set $\hat{\phi}_{k}(x)=\left(L_{k}(x)-L_{k+1}(x)\right) e^{-x / 2}$ so that $\hat{X}_{N}=$ $\operatorname{span}\left\{\hat{\phi}_{0}, \hat{\phi}_{1}, \ldots, \hat{\phi}_{N-1}\right\}$. Let $\hat{I}_{N}: C\left(R_{+}\right) \rightarrow \hat{P}_{N}$ be the interpolation operator based on the Laguerre-Gauss-Radau points $\left\{x_{j}\right\}_{j=0}^{N}$, and

$$
\begin{aligned}
& u_{N}=\sum_{k=0}^{N-1} \tilde{u}_{k} \hat{\phi}_{k}(x), \quad \bar{u}=\left(\tilde{u}_{0}, \tilde{u}_{1}, \ldots, \tilde{u}_{N-1}\right)^{T}, \\
& f_{i}=\left(\hat{I}_{N} f, \hat{\phi}_{i}\right), \quad \bar{f}=\left(f_{0}, f_{1}, \ldots, f_{N-1}\right)^{T}, \\
& s_{i j}=\left(\hat{\phi}_{j}^{\prime}, \hat{\phi}_{i}^{\prime}\right), \quad S=\left(s_{i j}\right)_{i, j=0,1, \ldots, N-1}, \\
& c_{i j}=\left(\hat{\phi}_{j}, \hat{\phi}_{i}\right), \quad C=\left(c_{i j}\right)_{i, j=0,1, \ldots, N-1} .
\end{aligned}
$$

It is straightforward to verify that $C$ is a symmetric tridiagonal matrix and that $S=I-\frac{1}{4} C$. Thus, the formulation (3.8) is reduced to the following linear system:

$$
\left(I+\left(\alpha-\frac{1}{4}\right) C\right) \bar{u}=\bar{f} .
$$

Remark 3.1. $\alpha>0$ is a necessary condition for the well-posedness of (3.7) and (3.8). One can also verifies that given $\alpha>0$, the tridiagonal matrix in (3.10) is strictly diagonally dominant and its condition number is uniformed bounded for all $N$.

In Figure 3.1, we compare the exact solutions in Examples 1 and 2 with approximate solutions obtained by the Galerkin approximation using Laguerre functions. To illustrate the rate of the convergence, we plot in Figure 3.2 the maximum errors at the Laguerre-Gauss-Radau points and the discrete $H^{1}\left(R_{+}\right)$errors. For Example 1, Theorem 3.1 asserts that the approximate solution will converge faster than any algebraic power. This is confirmed by Figure 3.2(A) which indicates that for Example 1, 

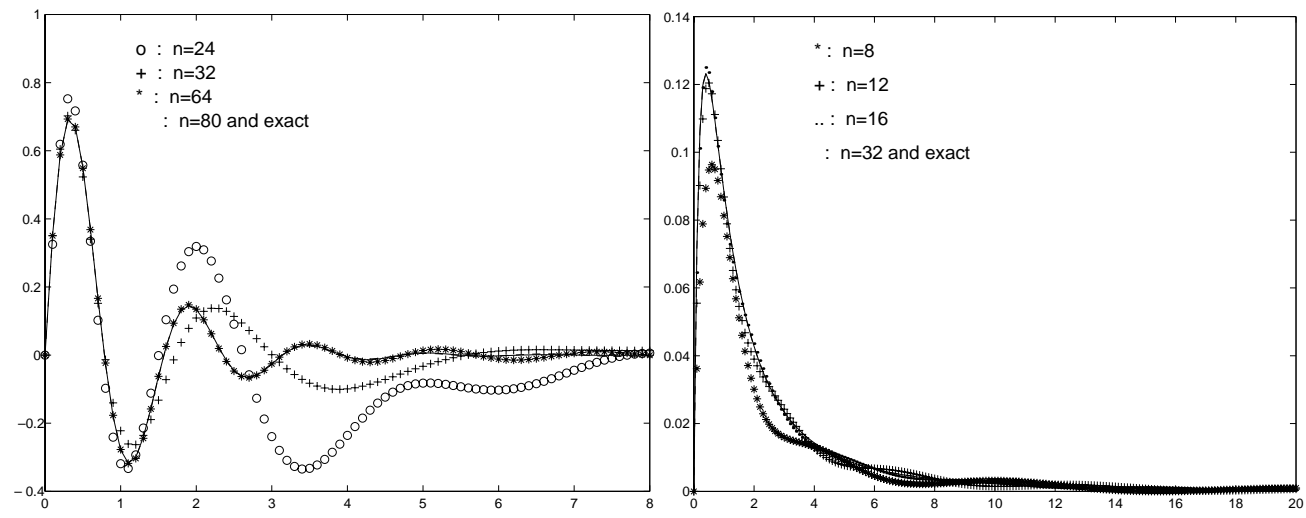

FIG. 3.1. Galerkin approximation using Laguerre functions: Example 1 with $k=4$ is on the left and Example 2 with $h=3.5$ is on the right.
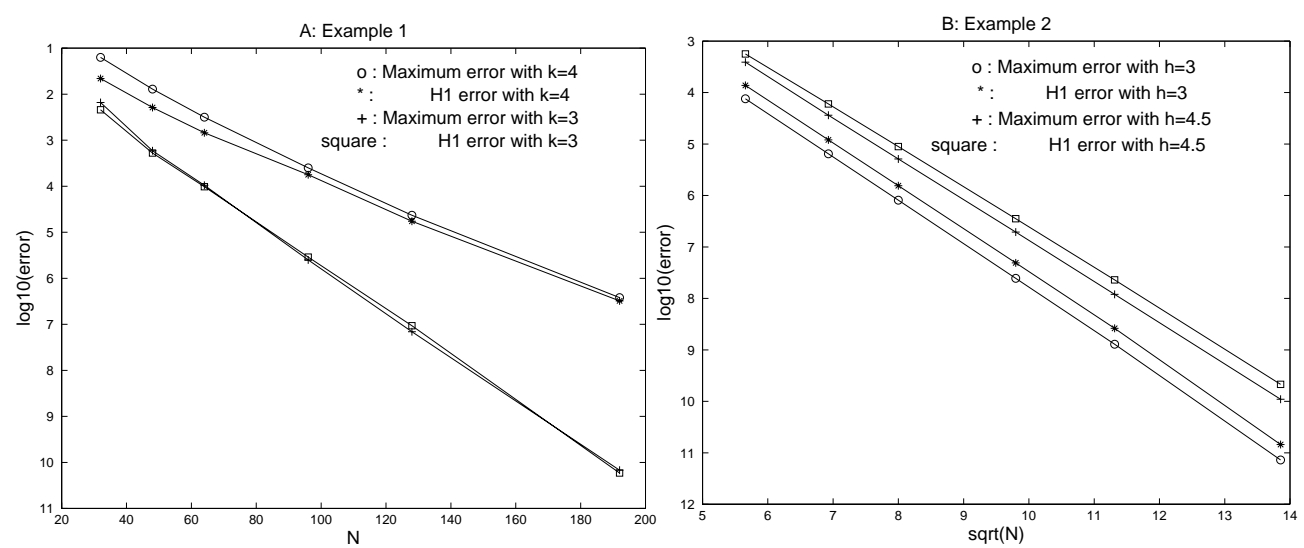

FIG. 3.2. Convergence rates of the Galerkin approximation using Laguerre functions.

the maximum and discrete $H^{1}$ errors behave like $e^{-c N}$. For Example 2, Theorem 3.1 predicts only a convergence rate of no more than $h$. However, Figure 3.2(B) clearly indicates that both the maximum and discrete $H^{1}$ errors behave like $e^{-c \sqrt{N}}$. This exponential convergence, although somewhat unexpected, can be explained by the fact that the function has no essential singularity at infinity. How to improve the error estimate in Theorem 3.1 to reflect this exponential convergence is still an open question.

For solutions which decay algebraically and have essential singularity at infinity, the convergence rate of its expansion in Laguerre functions will only be algebraic. A typical example of this is given below.

Example 3. $u(x)=\frac{\sin k x}{(1+x)^{h}}$ (algebraic decay with essential singularity at infinity).

For this example, Theorem 3.1 predicts a convergence rate of no more than $h$ in the $H^{1}$ norm. In Figure 3.3, we plot the discrete $H^{1}$ and $L^{2}$ errors for Example 3 for various $N$ with $k=3$ and $h=3$, 4 . It is clear that in all cases, the errors converge at 


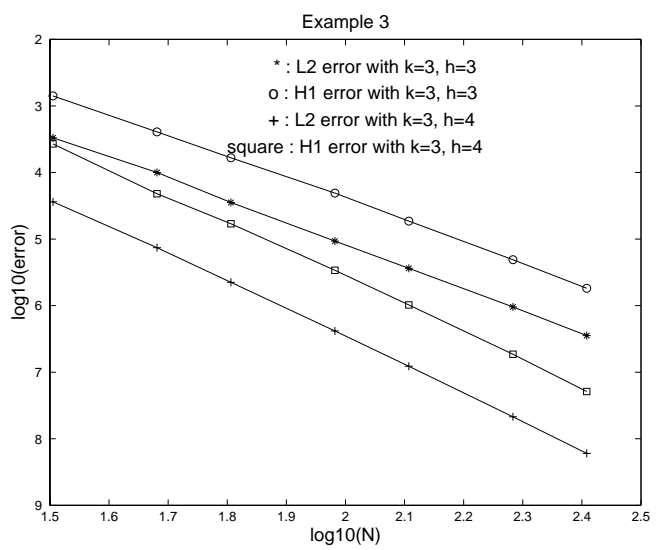

FIG. 3.3. Convergence rates of the Galerkin approximation using Laguerre functions.

a fixed algebraic rate. In fact, the observed convergence rates for both the discrete $H^{1}$ and $L^{2}$ errors are about $h$. This is consistent with the fact that the usual duality argument does not lead to improved error estimate in $L^{2}$ norm in this case.

3.3. Galerkin method with numerical integration. Although the above Galerkin method is very accurate and efficient for problems with constants coefficients and certain polynomial or rational coefficients, it is not directly applicable to problems with general coefficients. For the latter, the so-called Galerkin method with numerical integration should be used. Consider the model equation

$$
-\left(a(x) u_{x}\right)_{x}+b(x) u=f, \quad x \in R_{+} ; u(0)=0, \lim _{x \rightarrow+\infty} u(x)=0,
$$

where $a(x), b(x)>0 \forall x \in R_{+}$. The variational formulation for (3.11) is the following: Find $u \in H_{0}^{1}\left(R_{+}\right)$such that

$$
\left(a u_{x}, v_{x}\right)+(b u, v)=(f, v) \forall v \in H_{0}^{1}\left(R_{+}\right) .
$$

Let us define a discrete inner product

$$
(f, g)_{N}:=\sum_{i=0}^{N} f\left(x_{i}\right) g\left(x_{i}\right) \hat{\omega}_{i}
$$

associated with the Gauss-Radau quadrature (A.3) in the appendix, where $\left\{\hat{\omega}_{i}\right\}_{i=0}^{N}$ are given in (A.2). Then we can replace the continuous inner product $(\cdot, \cdot)$ in $(3.8)$ by the discrete one, leading to the following Laguerre-Galerkin method with numerical integration: Find $u_{N} \in \hat{X}_{N}$ such that

$$
\left(a u_{N}^{\prime}, v^{\prime}\right)_{N}+\left(b u_{N}, v\right)_{N}=(f, v)_{N} \forall v \in \hat{X}_{N}
$$

Let $\left\{h_{j}(x)\right\}_{j=0, \ldots, N}$ be the Lagrange interpolation polynomials in $P_{N}$ associated with the Gauss-Radau points $\left\{x_{j}\right\}_{j=0, \ldots, N}$. It is easy to show that

$$
h_{j}(x)=-\frac{x L_{N+1}^{\prime}(x)}{(N+1) L_{N+1}\left(x_{j}\right)\left(x-x_{j}\right)} ;
$$




$$
h_{j}^{\prime}\left(x_{i}\right)= \begin{cases}\frac{L_{N+1}\left(x_{i}\right)}{L_{N+1}\left(x_{j}\right)\left(x-x_{j}\right)}, & i \neq j, \\ \frac{1}{2}, & i=j \neq 0, \\ -\frac{N}{2}, & i=j=0 .\end{cases}
$$

Setting $\hat{h}_{j}(x)=h_{j}(x) \frac{e^{-x / 2}}{e^{-x_{j} / 2}}$, which is the Lagrange interpolating function in $\hat{P}_{N}$, so that

$$
\hat{h}_{j}(x) \in \hat{P}_{N}, \hat{h}_{j}\left(x_{i}\right)=\delta_{i j}, \text { and } \hat{X}_{N}=\operatorname{span}\left\{\hat{h}_{j}: j=1, \ldots, N\right\} .
$$

Thus any function $g$ in $\hat{X}_{N}$ can be written as $g(x)=\sum_{j=1}^{N} g\left(x_{j}\right) \hat{h}_{j}(x)$. We derive easily from (3.16) that

$$
\hat{h}_{j}^{\prime}\left(x_{i}\right)=\frac{e^{-x_{i} / 2}}{e^{-x_{j} / 2}}\left(h_{j}^{\prime}\left(x_{i}\right)-\frac{1}{2} \delta_{i j}\right)= \begin{cases}\frac{\hat{L}_{N+1}\left(x_{i}\right)}{\hat{L}_{N+1}\left(x_{j}\right)\left(x-x_{j}\right)}, & i \neq j, \\ 0, & i=j \neq 0, \\ -\frac{N+1}{2}, & i=j=0 .\end{cases}
$$

Hence $\hat{h}_{j}^{\prime}\left(x_{i}\right)$ can be computed in a stable way by using the procedure described in the appendix.

Let us denote

$$
\begin{aligned}
& \bar{u}=\left(u_{N}\left(x_{1}\right), \ldots, u_{N}\left(x_{N}\right)\right)^{T}, \bar{f}=\left(f\left(x_{1}\right), \ldots, f\left(x_{N}\right)\right)^{T}, \\
& s_{i j}=\left(a \hat{h}_{j}^{\prime}, \hat{h}_{i}^{\prime}\right)_{N}+\left(b h_{j}, h_{i}\right)_{N}=\sum_{k=0}^{N} \hat{h}_{j}^{\prime}\left(x_{k}\right) \hat{h}_{i}\left(x_{k}\right) a\left(x_{k}\right) \hat{\omega}_{k}+b\left(x_{i}\right) \hat{\omega}_{i} \delta_{i j}, \\
& W=\operatorname{diag}\left(\hat{\omega}_{1}, \ldots, \hat{\omega}_{N}\right), S=\left(s_{i j}\right)_{i, j=1, \ldots, N} .
\end{aligned}
$$

Thanks to (A.3), one verifies by using integration by parts that (3.14) is reduced to the following linear system:

$$
S \bar{u}=W \bar{f} .
$$

The linear system (3.19) is symmetric positive definite, but it is obviously full.

Remark 3.2. In the case of the Dirichlet boundary condition, the usual collocation method is the following: Find $u_{N} \in \hat{X}_{N}$ such that

$$
\left[-\left(a\left(u_{N}\right)_{x}\right)_{x}+b u_{N}\right]\left(x_{i}\right)=f\left(x_{i}\right), i=1,2, \ldots, N,
$$

is equivalent to the Galerkin method with numerical integration. In fact, the linear system associated with (3.20) is

$$
W^{-1} S \bar{u}=\bar{f} .
$$

However, for Neumann or mixed boundary conditions, the usual collocation method and the Galerkin method with numerical integration are not equivalent. As a rule of thumb, it is always preferable to us the Galerkin method with numerical integration instead of the usual collocation method, since (i) it is easier to analyze and usually leads to optimal error estimates; (ii) it leads to symmetric linear system for self-adjoint problems; and (iii) it leads to a linear system with smaller condition numbers (see Table 1 below).

We have listed in Table 1 the condition numbers of $S$ and $W^{-1} S$ in the model case: $a(x)=b(x)=1$. It is clear that condition numbers of $S$ in (3.19) grow like $O\left(N^{3 / 2}\right)$ while that of $W^{-1} S$ in (3.21) grows like $O\left(N^{2}\right)$. Hence, effective preconditioning is desirable for $N$ large, especially when the problems are multidimensional. We shall discuss two preconditioners below. 
TABLE 1

Condition numbers with $a(x)=b(x)=1$.

\begin{tabular}{|c|ccccccc|}
\hline$N$ & 8 & 16 & 32 & 64 & 128 & 256 & 512 \\
\hline Cond \# of (3.19) & 4.72 & 8.75 & 23.28 & 64.23 & 179.71 & 505.75 & 1427.07 \\
\hline Cond \# of (3.21) & 9.09 & 30.47 & 112.68 & 434.95 & 1710.87 & 6788.25 & 27045.16 \\
\hline Cond \# (3.26) & 1.60 & 1.98 & 2.35 & 2.75 & 3.25 & 3.92 & 4.86 \\
\hline Cond \# (3.27) & 2.50 & 2.66 & 2.77 & 2.84 & 2.89 & 2.93 & 2.95 \\
\hline
\end{tabular}

3.3.1. Preconditioning in frequency space. If both $a(x)$ and $b(x)$ are bounded from above and below by positive numbers, the problem (3.11) is spectrally equivalent to the constant coefficient problem (3.7) for any $\alpha>0$, especially for $\alpha=\frac{1}{4}$ when the matrix associated with the Laguerre-Galerkin approximation (3.10) becomes the identity matrix. Hence we expect that the condition number of the equivalent linear system, obtained from (3.14) by using the basis functions $\left\{\hat{\phi}_{k}\right\}_{k=0,1, \ldots, N-1}$, is also uniformly bounded. More precisely, let $\bar{u}$ and $\bar{f}$ be defined in (3.9), and define

$$
r_{j k}=\left(a \hat{\phi}_{k}^{\prime}, \hat{\phi}_{j}^{\prime}\right)_{N}+\left(b \hat{\phi}_{k}, \hat{\phi}_{j}\right)_{N}, \quad R=\left(r_{j k}\right)_{j=1, \ldots, N ; k=0,1, \ldots, N-1} .
$$

Then, by taking $v=\hat{\phi}_{k}$ in (3.14), we can rewrite it as

$$
R \bar{u}=\bar{f} .
$$

Lemma 3.4. Assuming $a(x), b(x)>0$ for $x \in R_{+}$, then the matrix $R$ is symmetric positive definite and its condition number is bounded by

$$
C_{a, b}(N):=\frac{\max \left\{\max _{j=0,1, \ldots, N} a\left(x_{j}\right), 4 \max _{j=0,1, \ldots, N} b\left(x_{j}\right)\right\}}{\min \left\{\min _{j=0,1, \ldots, N} a\left(x_{j}\right), 4 \min _{j=0,1, \ldots, N} b\left(x_{j}\right)\right\}} .
$$

Proof. For any $\bar{v}=\left(\tilde{v}_{0}, \tilde{v}_{1}, \ldots, \tilde{v}_{N-1}\right)^{T}$, we set $v(x)=\sum_{k=0}^{N-1} \tilde{v}_{k} \hat{\phi}_{k}(x)$. Thanks to (A.3), we have

$$
\begin{aligned}
(R \bar{v}, \bar{v})_{l^{2}} & =\left(a v^{\prime}, v^{\prime}\right)_{N}+(b v, v)_{N} \leq \max _{j=0,1, \ldots, N} a\left(x_{j}\right)\left(v^{\prime}, v^{\prime}\right)+\max _{j=0,1, \ldots, N} b\left(x_{j}\right)(v, v) \\
& \leq \max \left\{\max _{j=0,1, \ldots, N} a\left(x_{j}\right), 4 \max _{j=0,1, \ldots, N} b\left(x_{j}\right)\right\}\left[\left(v^{\prime}, v^{\prime}\right)+\frac{1}{4}(v, v)\right]
\end{aligned}
$$

and

$$
\begin{aligned}
(R \bar{v}, \bar{v})_{l^{2}} & \geq \min _{j=0,1, \ldots, N} a\left(x_{j}\right)\left(v^{\prime}, v^{\prime}\right)+\min _{j=0,1, \ldots, N} b\left(x_{j}\right)(v, v) \\
& \geq \min \left\{\min _{j=0,1, \ldots, N} a\left(x_{j}\right), 4 \min _{j=0,1, \ldots, N} b\left(x_{j}\right)\right\}\left[\left(v^{\prime}, v^{\prime}\right)+\frac{1}{4}(v, v)\right] .
\end{aligned}
$$

We then conclude from the fact (see (3.10)) that

$$
\left(v^{\prime}, v^{\prime}\right)+\frac{1}{4}(v, v)=(\bar{v}, \bar{v})_{l^{2}} .
$$

Remark 3.3. In fact, no preconditioner is applied here for the one-dimensional problem since it is well conditioned in the frequency space. However, in the multidimensional cases the corresponding linear system will be ill conditioned even in 
the frequency space. However, one can use a constant-coefficient problem in the frequency space as a preconditioner which is optimal as long as the variable coefficients are bounded from above and below.

Hence one can directly use the conjugate gradient method to solve (3.23). This approach is very effective if $a(x)$ and $b(x)$ do not have large variations. Otherwise, a preconditioner based on the finite element/finite difference approximation in the physical space may be more appropriate.

3.3.2. Preconditioning in physical space. A more traditional preconditioner can be built using a finite element approximation for (3.11) on the Laguerre-GaussRadau points. The idea of using finite difference/finite element approximation to build preconditioners for the spectral-collocation system was first introduced in [17] and has been used extensively and successfully for problems in finite domains. However, to the best of our knowledge, its application to problems in infinite domains has not been exploited. In order to build a preconditioner using the finite element approximation, we need to introduce an extra point $x_{N+1}$ in $\left(x_{N},+\infty\right)$. Let

$$
V_{N}=\left\{u \in H_{0}^{1}\left(0, X_{N+1}\right):\left.u\right|_{\left[x_{i}, x_{i+1}\right]} \in P_{1}, i=0,1, \ldots, N\right\} .
$$

The finite element approximation of (3.11) in $V_{N}$ leads to a linear system of the form

$$
S_{f e} \bar{u}=M_{f e} \bar{f},
$$

where $S_{f e}$ and $M_{f e}$ are, respectively, the stiffness and mass matrices associated with $V_{N}$ for (3.11), and $\bar{u}$ and $\bar{f}$ are the same as in (3.19). Hence, instead of solving the ill-conditioned system (3.21), it is natural to solve the equivalent preconditioned system

$$
S_{f e}^{-1} M_{f e} W^{-1} S \bar{u}=S_{f e}^{-1} M_{f e} \bar{f} .
$$

Our experiments seem to indicate that the exact location of $x_{N+1}$ is insignificant. Therefore we have taken $x_{N+1}=x_{N}+\left(x_{N}-x_{N-1}\right)$. In the third row of Table 1, the condition numbers of $S_{f e}^{-1} M_{f e} W^{-1} S$ are listed in the case of $a(x)=b(x)=1$. Unfortunately, unlike in the case of finite intervals where finite element/finite difference approximations provide optimal preconditioners for the spectral approximation, the condition number here grows like $O\left(N^{\beta}\right)$ for $\beta \sim 0.3$, although it remains very small in practical ranges (less than 5 for $N$ up to 512 ).

We can also view $S_{f e}$ as an approximation of $S$ and consider instead the preconditioned system

$$
S_{f e}^{-1} S \bar{u}=S_{f e}^{-1} \bar{f} .
$$

In this case, the exact location of $x_{N+1}$ does play an important role. Our experiment indicates that $x_{N+1}=x_{N}+\left(x_{N}-x_{N-1}\right)$ is a suitable choice.

The condition numbers of $S_{f e}^{-1} S$ are listed in the fourth row of Table 1. It is clear that they are uniformly bounded. Furthermore, since both $S_{f e}$ and $S$ are symmetric positive definite, (3.27) can be solved by using the standard preconditioned conjugate gradient method.

In order to compare the robustness with respect to coefficient variations of the systems (3.23) and (3.27), we have considered two sets of coefficients: (i) $a(x)=$ $1+\sin ^{2}(6 x)$ and $b(x)=1$ such that the variations of $a$ and $b$ are relatively small $\left(C_{a, b}(N) \leq 4\right)$, and (ii) $a(x)=((x+1) /(x+6))^{6}$ and $b(x)=1$ such that the variations 
TABLE 2

Condition numbers with variable $a(x)$ and $b(x)$.

\begin{tabular}{|c|c|cccccc|}
\hline Case & $N$ & 8 & 16 & 32 & 64 & 128 & 256 \\
\hline (i) & Cond \# of (3.23) & 3.35 & 3.68 & 3.84 & 3.93 & 3.97 & 3.99 \\
\hline (i) & Cond \# (3.27) & 2.91 & 2.74 & 3.28 & 3.80 & 3.87 & 4.19 \\
\hline (ii) & Cond \# of (3.23) & 31.76 & 113.95 & 426.58 & 1632.39 & 6225.64 & 22334.10 \\
\hline (ii) & Cond \# of (3.27) & 2.78 & 3.05 & 3.48 & 4.29 & 5.96 & 9.25 \\
\hline
\end{tabular}

of $a$ and $b$ are relatively large $\left(C_{a, b}(N) \leq 4 * 6^{6}\right)$. The condition numbers of (3.23) and (3.27) are listed in Table 2.

It is clear that (3.23) is effective only if $C_{a, b}(N)$ in Lemma 3.4 is relatively small. On the other hand, (3.27) is quite robust with respect to the coefficient variations. Thus (3.27) should be used in practice in the case of large coefficient variations.

3.4. Scaling. Although the Laguerre-spectral methods presented above enjoy a theoretical spectral convergence rate, the actual error decays considerably slower than the Chebyshev- or Legendre-spectral method for similar problems in finite intervals. The poor resolution property of Laguerre polynomials/functions, which was pointed out by Gottlieb and Orszag in [10], is one of the main reasons why Laguerre polynomials/functions are rarely used in practice. However, similarly as in [23] for the Hermite spectral method, the resolution of Laguerre functions can be greatly improved by using a proper scaling factor.

The main factor responsible for the poor resolution of Laguerre polynomials and Laguerre functions is that usually a significant portion of the Laguerre-Gauss-Radau points is located outside of the interested interval (see Figure 3.4, which illustrates locations of the Laguerre-Gauss-Radau points). For example, $u(x)=\sin k x e^{-x} \leq$ $10^{-8}$ for $x>18$. Therefore all the collocation points which are greater than 18 are essentially wasted. Thus it makes sense to scale the function so that all the effective collocation points are inside the interested interval. More precisely, we can proceed as follows: Given an accuracy threshold $\varepsilon$, we estimate an $M$ such that $|u(x)| \leq \varepsilon$ for $x>M$. Then we set the scaling factor $\beta_{N}=x_{N}^{(N)} / M$ where $x_{N}^{(N)}$ is the largest Laguerre-Gauss-Lobatto point, and instead of solving (3.7), we solve the following scaled equation with the new variable $y=\beta_{N} x$ :

$$
\alpha v-\beta_{N}^{2} v_{y y}=g(y) ; v(0)=0, \lim _{y \rightarrow+\infty} u(y)=0,
$$

where $v(y)=u\left(\beta_{N} x\right)$ and $g(y)=f\left(\beta_{N} x\right)$. Thus the effective collocation points $x_{j}=y_{j} / \beta_{N}\left(\left\{y_{j}\right\}_{j=0}^{N}\right.$ being the Laguerre-Gauss-Lobatto points) are all located in $[0, M]$. In Figure 3.4, the approximations of Example 3 with $k=10$ and $h=5$ using the Laguerre-Galerkin method with a scaling factor $=15$ and without scaling are plotted against the exact solution. Notice that if no scaling is used, the approximation with $N=128$ still exhibits an observable error, while the approximation with a scaling factor $=15$ using only 32 modes is virtually indistinguishable with the exact solution. This simple example demonstrates that a proper scaling will greatly enhance the resolution capabilities of the Laguerre functions and make the Laguerre functions a viable alternative to the rational polynomials studied in $[11,3]$. 


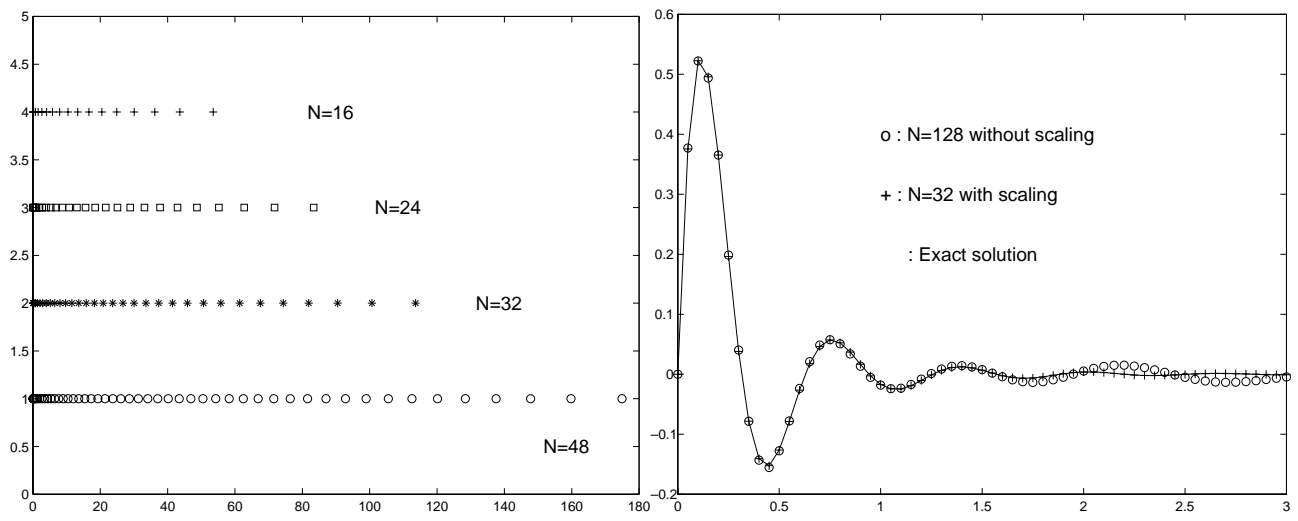

FIG. 3.4. Locations of the Laguerre-Gauss-Radau points and effects of scaling.

\section{Miscellaneous extensions.}

4.1. General boundary conditions. More general boundary conditions of the form

$$
a u(0)-b u^{\prime}(0)=c,
$$

with $b \neq 0$ ( $b=0$ reduces to the simpler Dirichlet case) and $a b \geq 0$ to ensure the ellipticity, can be easily handled as follows. First, the nonhomogeneity can be taken care of by subtracting $u_{b}(x):=c /(a+b / 2) e^{-x / 2}$ from the solution, thus leading to the following homogeneous problem: Find $u=\tilde{u}+u_{b}$ such that

$$
\alpha \tilde{u}-\tilde{u}_{x x}=f-\left(\alpha-\frac{1}{4}\right) u_{b}:=\tilde{f} ; a \tilde{u}(0)-b \tilde{u}^{\prime}(0)=0 .
$$

The corresponding variational formulation is the following: Find $u=\tilde{u}+u_{b} \in H^{1}\left(R_{+}\right)$ such that

$$
\alpha(\tilde{u}, v)+\frac{a}{b} \tilde{u}(0) v(0)+\left(\tilde{u}_{x}, v_{x}\right)=(\tilde{f}, v) \forall v \in H^{1}\left(R_{+}\right) .
$$

Next, setting

$\tilde{\phi}_{k}(x)=\left(L_{k}(x)-a_{k} L_{k+1}(x)\right) e^{-x / 2}$ with $a_{k}=\left(a+k b+\frac{1}{2}\right) /\left(a+(k+1) b+\frac{1}{2}\right)$,

and

$$
\tilde{X}_{N}=\operatorname{span}\left\{\tilde{\phi}_{0}, \tilde{\phi}_{1}, \ldots, \tilde{\phi}_{N-1}\right\} .
$$

Thanks to $(2.2)$, we have $a \tilde{\phi}_{k}(0)+b \tilde{\phi}_{k}^{\prime}(0)=0$. Hence the Galerkin method for $(4.3)$ is the following:

Find $u_{N}=\tilde{u}_{N}+u_{b}$ with $\tilde{u}_{N} \in \tilde{X}_{N}$ such that

$$
\left(\tilde{u}_{N}^{\prime}, \tilde{v}^{\prime}\right)+\frac{a}{b} \tilde{u}_{N}(0) \tilde{v}(0)+\alpha\left(\tilde{u}_{N}, \tilde{v}\right)=(\tilde{f}, \tilde{v}) \forall \tilde{v} \in \tilde{X}_{N}
$$


It is clear that $\tilde{C}_{i j}:=\left(\tilde{\phi}_{j}, \tilde{\phi}_{i}\right)=0$ for $|i-j|>1$. One can also verify readily by integration by parts that

$$
\tilde{S}_{i j}:=\left(\tilde{\phi}_{j}^{\prime}, \tilde{\phi}_{i}^{\prime}\right)+\min \left(\frac{b}{a}, 0\right) \tilde{\phi}_{j}(0) \tilde{\phi}_{i}(0)=-\left(\tilde{\phi}_{j}^{\prime \prime}, \tilde{\phi}_{i}\right)=-\left(\tilde{\phi}_{j}, \tilde{\phi}_{i}^{\prime \prime}\right)
$$

which implies that $\tilde{S}_{i j}=0$ for $|i-j|>1$. Hence the matrix $\tilde{S}+\alpha \tilde{C}$ associated with (4.4) is again tridiagonal. Note that in order to obtain a sparse linear system, it is necessary to treat the boundary condition (4.1) as an "essential" boundary condition in (4.4).

4.2. Fourth-order equations. Consider the fourth-order model problem

$$
\begin{aligned}
& \alpha_{1} u-\alpha_{2} u_{x x}+u_{x x x x}=f, \quad x \in R_{+}, \\
& u(0)=u_{x}(0)=0, \quad \lim _{x \rightarrow+\infty} u(x)=0, \quad \lim _{x \rightarrow+\infty} u_{x}(x)=0 .
\end{aligned}
$$

Let $H_{0}^{2}\left(R_{+}\right)=\left\{u \in H^{2}\left(R_{+}\right): u(0)=u_{x}(0)=0\right\}$. The variational formulation for (4.6) is the following:

Find $u \in H_{0}^{2}\left(R_{+}\right)$such that

$$
\alpha_{1}(u, v)+\alpha_{2}\left(u_{x}, v_{x}\right)+\left(u_{x x}, v_{x x}\right)=(f, v) \forall v \in H_{0}^{2}\left(R_{+}\right) .
$$

Let us denote $\hat{W}_{N}=\left\{u \in \hat{P}_{N}: u(0)=u_{x}(0)=0\right\}$. Then the Laguerre-Galerkin approximation for (4.7) is the following:

Find $u_{N} \in \hat{W}_{N}$ such that

$$
\alpha_{1}\left(u_{N}, v\right)+\alpha_{2}\left(u_{N}^{\prime}, v^{\prime}\right)+\left(u_{N}^{\prime \prime}, v^{\prime \prime}\right)=(f, v) \forall v \in \hat{W}_{N} .
$$

One verifies easily from $(2.2)$ that $\hat{\psi}_{k}(x)=\left(L_{k}(x)-2 L_{k+1}(x)+L_{k+2}(x)\right) e^{-x / 2} \in \hat{W}_{N}$ and $\hat{W}_{N}=\operatorname{span}\left\{\hat{\psi}_{0}, \hat{\psi}_{1}, \ldots, \hat{\psi}_{N-2}\right\}$. Hence, setting

$$
\begin{aligned}
& a_{k j}=\left(\hat{\psi}_{j}^{\prime \prime}, \hat{\psi}_{k}^{\prime \prime}\right), A=\left(a_{k j}\right)_{k, j=0,1, \ldots, N-2}, \\
& b_{k j}=\left(\hat{\psi}_{j}^{\prime}, \hat{\psi}_{k}^{\prime}\right), B=\left(b_{k j}\right)_{k, j=0,1, \ldots, N-2}, \\
& c_{k j}=\left(\hat{\psi}_{j}, \hat{\psi}_{k}\right), C=\left(c_{k j}\right)_{k, j=0,1, \ldots, N-2}, \\
& \tilde{f}_{k}=\left(\hat{I}_{N} f, \hat{\psi}_{k}\right), \bar{f}=\left(\tilde{f}_{0}, \tilde{f}_{1}, \ldots, \tilde{f}_{N-2}\right), \\
& u_{N}=\sum_{k=0}^{N-2} \tilde{u}_{k} \hat{\psi}_{k}, \bar{u}=\left(\tilde{u}_{0}, \tilde{u}_{1}, \ldots, \tilde{u}_{N-2}\right),
\end{aligned}
$$

we find that (4.8) (with $f$ replaced by $\hat{I}_{N} f$ for practical implementations) reduces to

$$
\left(\alpha_{1} C+\alpha_{2} B+A\right) \bar{u}=\bar{f} .
$$

One verifies that $A, B$, and $C$ are all symmetric pentadiagonal and their entries can be easily computed. Hence (4.8) can be efficiently solved.

The error analysis for the Laguerre approximation of the fourth-order problem is quite similar to that of the second-order problem. Hence, we shall sketch only some corresponding results below.

Let us first define an orthogonal projection operator $\pi_{N}^{2}: H_{\omega}^{2}\left(R_{+}\right) \rightarrow P_{N}$ by

$$
\left(\left(u-\pi_{N}^{2} u\right)^{\prime \prime}, v_{N}^{\prime \prime}\right)_{\omega}+\left(\left(u-\pi_{N}^{2} u\right)^{\prime}, v_{N}^{\prime}\right)_{\omega}+\left(\left(u-\pi_{N}^{2} u\right), v_{N}\right)_{\omega}=0 \quad \forall v_{N} \in P_{N} .
$$


Setting $H_{0, \omega}^{2}\left(R_{+}\right)=\left\{u \in H_{\omega}^{2}\left(R_{+}\right): u(0)=u^{\prime}(0)=0\right\}$ and $W_{N}=\left\{u \in P_{N}: u(0)=\right.$ $\left.u^{\prime}(0)=0\right\}$, we define $\pi_{N}^{2,0}: H_{0, \omega}^{2}\left(R_{+}\right) \rightarrow W_{N}$ by

$$
\left(\left(u-\pi_{N}^{2,0} u\right)^{\prime \prime}, v_{N}^{\prime \prime}\right)_{\omega}=0 \quad \forall v_{N} \in W_{N} .
$$

Lemma 4.1. Let $r \geq 2$ and $\beta$ be the largest integer such that $\beta<r-1$; then

$\left\|u-\pi_{N}^{2} u\right\|_{2, \omega} \lesssim N^{1-\frac{r}{2}}\left\|u_{x x}\right\|_{r-2, \omega, \beta} \quad \forall u$ s.t. $u_{x x} \in H_{\omega, \beta}^{r-2}\left(R_{+}\right)$,

$\left\|u-\pi_{N}^{2,0} u\right\|_{2, \omega} \lesssim N^{1-\frac{r}{2}}\left\|u_{x x}\right\|_{r-2, \omega, \beta} \quad \forall u$ s.t. $u_{x x} \in H_{\omega, \beta}^{r-2}\left(R_{+}\right), u \in H_{0, \omega}^{2}\left(R_{+}\right)$.

Proof. Given $u \in H_{\omega, \beta}^{r}\left(R_{+}\right)$, we set

$$
u_{N}=u(0)+u^{\prime}(0) x+\int_{0}^{x} \int_{0}^{z} \pi_{N-2} u^{\prime \prime}(y) d y d z,
$$

where $\pi_{N-2}$ is the orthogonal projection operator in $L_{\omega}^{2}\left(R_{+}\right)$defined in section 2 . Then we have

$$
u_{N} \in P_{N}, u_{N}(0)=u(0), u_{N}^{\prime}(0)=u^{\prime}(0), u_{N}^{\prime \prime}(x)=\pi_{N-2} u^{\prime \prime}(x) .
$$

Hence, $u-u_{N} \in H_{0, \omega}^{2}\left(R_{+}\right)$, and thanks to Lemmas 2.1 and 2.2,

$$
\begin{aligned}
\left\|u-\pi_{N}^{2} u\right\|_{2, \omega} & \leq\left\|u-u_{N}\right\|_{2, \omega} \leq\left|u-u_{N}\right|_{2, \omega} \\
& =\left\|u^{\prime \prime}-\pi_{N-2} u^{\prime \prime}\right\|_{\omega} \lesssim N^{1-\frac{r}{2}}\left\|u_{x x}\right\|_{r-2, \omega, \beta} .
\end{aligned}
$$

The second inequality can be proved similarly. Indeed, given $u \in H_{0, \omega}^{2}\left(R_{+}\right)$, we set

$$
u_{N}=\int_{0}^{x} \int_{0}^{z} \pi_{N-2} u^{\prime \prime}(y) d y d z
$$

Then we have $u_{N} \in W_{N}$ and $u_{N}^{\prime \prime}(x)=\pi_{N-2} u^{\prime \prime}(x)$. Hence

$$
\begin{aligned}
\left\|u-\pi_{N}^{2,0} u\right\|_{2, \omega} & \leq\left\|u-u_{N}\right\|_{2, \omega} \leq\left|u-u_{N}\right|_{2, \omega} \\
& =\left\|u^{\prime \prime}-\pi_{N-2} u^{\prime \prime}\right\|_{\omega} \lesssim N^{1-\frac{r}{2}}\left\|u_{x x}\right\|_{r-2, \omega, \beta} .
\end{aligned}
$$

Now we define $\hat{\pi}_{N}^{2,0}: H^{2}\left(R_{+}\right) \rightarrow \hat{W}_{N}$ by

$$
\hat{\pi}_{N}^{2,0} u=e^{-x / 2} \pi_{N}^{2,0}\left(u e^{x / 2}\right) \forall u \in H^{2}\left(R_{+}\right) .
$$

LEMMA 4.2.

$$
\left(\left(u-\hat{\pi}_{N}^{2,0} u\right)^{\prime \prime}, v_{N}^{\prime \prime}\right)+\frac{1}{2}\left(\left(u-\hat{\pi}_{N}^{2,0} u\right)^{\prime}, v_{N}^{\prime}\right)+\frac{1}{16}\left(u-\hat{\pi}_{N}^{2,0} u, v_{N}\right)=0 \forall v_{N} \in \hat{W}_{N},
$$

and

$$
\left\|u-\hat{\pi}_{N}^{2,0} u\right\|_{2} \lesssim N^{1-\frac{r}{2}}\left\|u_{x x}+u_{x}+\frac{1}{4} u\right\|_{r-2, \beta},
$$

where $r \geq 2$ and $\beta$ is the largest integer for which $\beta<r-1$. 
Proof. The first identity can be proved by straightforward computations.

Thanks to Lemmas 4.1 and 3.1, we have

$$
\begin{aligned}
\left\|u-\hat{\pi}_{N}^{2,0} u\right\|_{2} & =\left\|u e^{x / 2}-\pi_{N}^{2,0}\left(u e^{x / 2}\right)\right\|_{2, \omega} \lesssim N^{1-\frac{r}{2}}\left\|\left(u e^{x / 2}\right)_{x x}\right\|_{r-2, \omega, \beta} \\
& =N^{1-\frac{r}{2}}\left\|u_{x x}+u_{x}+\frac{1}{4} u\right\|_{r-2, \beta} .
\end{aligned}
$$

We are now in position to prove the following theorem.

THEOREM 4.1. Given $\alpha_{1}>0$ and $\alpha_{2}>0$, let $u$ and $u_{N}$ be, respectively, the solutions of (4.7) and (4.8). Let $r \geq 2$ and $\beta$ be the largest integer for which $\beta<r-1$. Then

$$
\left\|u-u_{N}\right\|_{2} \lesssim N^{1-\frac{r}{2}}\left\|u_{x x}+u_{x}+\frac{1}{4} u\right\|_{r-2, \beta} .
$$

Proof. Let $e_{N}=u_{N}-\hat{\pi}_{N}^{2,0} u$. We derive from (4.7), (4.8), and Lemma 4.2 that

$$
\begin{aligned}
\alpha_{1}\left(e_{N}, v\right)+\alpha_{2}\left(e_{N}^{\prime}, v^{\prime}\right)+\left(e_{N}^{\prime \prime}, v^{\prime \prime}\right)= & \left(\alpha_{1}-\frac{1}{2}\right)\left(u-\hat{\pi}_{N}^{2,0} u, v\right) \\
& +\left(\alpha_{2}-\frac{1}{16}\right)\left(\left(u-\hat{\pi}_{N}^{2,0} u\right)^{\prime}, v^{\prime}\right) \forall v \in \hat{W}_{N} .
\end{aligned}
$$

The result follows from Lemma 4.2 by taking $v=e_{N}$ in the above relation.

4.3. Multidimensional unbounded domains. For problems in multidimensional unbounded domains, we can develop efficient spectral-Galerkin methods, using Laguerre functions combined with Chebyshev or Legendre or Fourier polynomials, in the same way as we do for problems in multidimensional bounded domains (see, for instance, $[18,19,20,21])$. We will describe only briefly two typical cases below.

4.3.1. $\Omega=\left\{x^{2}+y^{2}>1\right\}$. Using the polar transform $x=r \cos \theta, y=r \sin \theta$, the Helmholtz equation

$$
\alpha U-\Delta U=F, \quad(x, y) \in \Omega ;\left.\quad U\right|_{\partial \Omega}=0, \lim _{r=\sqrt{x^{2}+y^{2}} \rightarrow+\infty} U(x, y)=0
$$

becomes

$$
\begin{aligned}
& \alpha u-\frac{1}{r}\left(r u_{r}\right)_{r}-\frac{1}{r^{2}} u_{\theta \theta}=f,(r, \theta) \in(1,+\infty) \times(0,2 \pi), \\
& u(1, \theta)=0, u \text { periodic in } \theta, \lim _{r \rightarrow+\infty} u(r, \theta)=0,
\end{aligned}
$$

where $u(r, \theta)=U(x, y)$ and $f(r, \theta)=F(x, y)$. Writing $u=\sum_{m=-\infty}^{+\infty} u_{m}(r) e^{i m \theta}$, reduces to a sequence of one-dimensional equations:

$$
\begin{aligned}
& \alpha u_{m}-\frac{1}{r} d_{r}\left(r d_{r}\left(u_{m}\right)\right)+\frac{m^{2}}{r^{2}} u_{m}=f_{m}, r \in(1,+\infty), \\
& u_{m}(1)=0, \lim _{r \rightarrow+\infty} u_{m}(r)=0 .
\end{aligned}
$$

Setting $r=t+1, w_{m}(t)=u_{m}(t+1)$ and $g_{m}(t)=f_{m}(t+1)$, we can approximate (4.15) by the following Laguerre-Galerkin method:

Find $w_{N}^{(m)} \in \hat{X}_{N}$ such that

$$
\begin{aligned}
& \left((t+1) d_{t} w_{N}^{(m)}, d_{t}((t+1) v)\right)+\alpha\left((t+1)^{2} w_{N}^{(m)}, v\right)+m^{2}\left(w_{N}^{(m)}, v\right) \\
= & \left(\hat{I}_{N} g_{m},(t+1)^{2} v\right) \forall v \in \hat{X}_{N} .
\end{aligned}
$$


Using the basis functions $\hat{\phi}_{k}(x)=\left(L_{k}(x)-L_{k+1}(x)\right) e^{-x / 2}$, we verify that the linear system associated with (4.16), although nonsymmetric, is again banded with bandwidth four so that it can be efficiently solved.

4.3.2. $\Omega=\boldsymbol{R}_{+} \times(-1,1)$. Consider again the Helmholtz equation

$$
\alpha u-\Delta u=f, \quad(x, y) \in \Omega=R_{+} \times(-1,1),\left.\quad u\right|_{\partial \Omega}=0, \lim _{x \rightarrow+\infty} u(x, y)=0 .
$$

Let $V_{M}=\left\{u \in P_{M}: u( \pm 1)=0\right\}$ and $\boldsymbol{W}_{N M}=\hat{X}_{N} \times V_{M}$. The Laguerre-ChebyshevGalerkin method for (4.17) is the following:

Find $u_{N M} \in \boldsymbol{W}_{N M}$ such that

$$
\begin{aligned}
\alpha \int_{\Omega} u_{N M} v \omega(y) d x d y & +\int_{\Omega} \nabla u_{N M} \cdot(\nabla v \omega(y)) d x d y \\
& =\int_{\Omega} \hat{I}_{N M} f v \omega(y) d x d y \quad \forall v \in \boldsymbol{W}_{N M},
\end{aligned}
$$

where $\omega(y)=\left(1-y^{2}\right)^{-\frac{1}{2}}$ is the Chebyshev weight, and $\hat{I}_{N M}: C(\Omega) \rightarrow \hat{P}_{N} \times P_{M}$ is the Laguerre-Chebyshev interpolation operator. Now setting $h_{k}(y)=T_{k}(y)-$ $T_{k+2}(y)$, where $T_{k}$ is the $k$ th degree Chebyshev polynomial, we have $h_{k} \in V_{M}$ for $k=0,1, \ldots, M-2$ and that

$$
\boldsymbol{W}_{N M}=\operatorname{span}\left\{\hat{\phi}_{k}(x) h_{j}(y): k=0,1, \ldots, N-1 ; j=0,1, \ldots, M-2\right\} .
$$

Then, denoting

$$
\begin{aligned}
& a_{k j}=\int_{-1}^{1} h_{j}^{\prime}(y)\left(h_{k}(y) \omega(y)\right)^{\prime} d y, A=\left(a_{k j}\right)_{k, j=0,1, \ldots, M-2}, \\
& b_{k j}=\int_{-1}^{1} h_{j}(y) h_{k}(y) \omega(y) d y, B=\left(b_{k j}\right)_{k, j=0,1, \ldots, M-2}, \\
& u_{N M}=\sum_{k=0}^{N-1} \sum_{j=0}^{M-2} \tilde{u}_{k j} \hat{\phi}_{k}(x) h_{j}(y), U=\left(\tilde{u}_{k j}\right)_{k=0,1, \ldots, N-1 ; j=0,1, \ldots, M-2,} \\
& \tilde{f}_{k j}=\int_{\Omega} \hat{I}_{N M} f v \omega(y) d x d y, F=\left(\tilde{f}_{k j}\right),
\end{aligned}
$$

we find that (4.18) reduces to the following matrix equation:

$$
\alpha C U B+S U B+C U A^{T}=F,
$$

where $C$ and $S$ are defined in (3.9). Let $(\Lambda, E)$ be the eigenpair of $C$ such that $C E=E \Lambda$ with $\Lambda=\operatorname{diag}\left(\lambda_{0}, \ldots, \lambda_{N-1}\right)$ and $E^{T} E=I$. Setting $U=E V$ and using the fact that $S=I-\frac{1}{4} C$, we can rewrite (4.20) as

$$
\left(\alpha-\frac{1}{4}\right) C E V B+E V B+C V A^{T}=F .
$$

Applying $E^{T}$ to the above equation and denoting $\bar{v}_{i}$ and $\bar{g}_{i}$ to be, respectively, the $i$ th row of $V$ and $E^{T} F$, we find that it reduces to

$$
\left[\lambda_{i} A+\left(1+\left(\alpha-\frac{1}{4}\right) \lambda_{i}\right) B\right] \bar{v}_{i}=\bar{g}_{i}, \quad i=0,1, \ldots, N-1 .
$$


It is shown in [19] that $A$ and $B$ have special structures such that the above equation for each $i$ can be solved in $O(M)$ operations. Furthermore, thanks to the special form of $C$, the two matrix-multiplication involving $E$ can be performed by using fast Fourier transforms just as we do in the finite difference fast Poisson solver (see, for instance, [4]). Exactly the same procedure applies if one chooses to use Legendre polynomials instead of Chebyshev polynomials in the $y$ direction.

5. Concluding remarks. We have presented stable and efficient spectral methods using Laguerre functions for model elliptic equations on regular unbounded domains. Our theoretical error estimates and numerical experiments indicate that

- from both the theoretical and practical points of view, the Laguerre polynomials are generally not suitable for practical implementations;

- with a proper scaling, the Galerkin method using Laguerre functions provides a very efficient yet very simple way for solving problems in unbounded domains;

- the Laguerre-Galerkin approximation converges exponentially for solutions which delay algebraicly but without essential singularity at infinity. However, only algebraic convergence is possible if the solution decays algebraicly but with an essential singularity at infinity.

In summary, as demonstrated in this paper and in [13] where a similar LaguerreGalerkin method is successfully used for the approximation of some nonlinear partial differential equations in semi-infinite intervals, we believe that properly formulated spectral methods using Laguerre functions are very valuable tools and viable alternatives to rational polynomials for problems in unbounded domains.

Appendix A. Stable discrete Laguerre transform. For a fixed integer $N$, the Laguerre-Gauss-Radau points $\left\{x_{i}\right\}_{i=0}^{N}$ are the roots of $x L_{N+1}^{\prime}(x)$. The associated Gauss-Radau quadrature is

$$
\int_{0}^{+\infty} f(x) g(x) e^{-x} d x=\sum_{i=0}^{N} f\left(x_{i}\right) g\left(x_{i}\right) \omega_{i} \forall f \in P_{K}, g \in P_{M} \text { s.t. } K+M \leq 2 N,
$$

where $\omega_{i}=\frac{1}{(N+1) L_{N}^{2}\left(x_{i}\right)}, i=0,1, \ldots, N$ which become exponentially small as $N$ increases. Therefore the usual discrete Laguerre transform based on (A.1) is not suitable for practical implementation. Instead, we should set

$$
\hat{\omega}_{j}=\omega_{j} e^{x_{j}}=\frac{1}{(N+1) \hat{L}_{N}^{2}\left(x_{j}\right)}
$$

and rewrite (A.1) as

$$
\int_{0}^{+\infty} f(x) g(x) d x=\sum_{i=0}^{N} f\left(x_{i}\right) g\left(x_{i}\right) \hat{\omega}_{i} \forall f \in \hat{P}_{K}, g \in \hat{P}_{M} \text { s.t. } K+M \leq 2 N .
$$

We will use this quadrature formula to derive the discrete transform using Laguerre functions.

Given $f \in C\left(R_{+}\right)$, the interpolation operator $\hat{I}_{N}: C\left(R_{+}\right) \longrightarrow \hat{P}_{N}$ based on Laguerre function is

$$
\hat{I}_{N} f=\sum_{n=0}^{N} a_{n} \hat{L}_{n}(x) \text { s.t. } \hat{I}_{N} f\left(x_{j}\right)=f\left(x_{j}\right), j=0,1, \ldots, N .
$$


Thanks to (3.1) and (A.3), $\left\{a_{n}\right\}$ can be determined by

$$
a_{n}=\int_{0}^{+\infty} \hat{I}_{N} f \hat{L}_{n}(x) d x=\sum_{j=0}^{N} f\left(x_{j}\right) \hat{L}_{n}\left(x_{j}\right) \hat{\omega}_{j} .
$$

Hence, in order to perform the (forward and backward) discrete transforms, we will need to be able to compute $\left\{\hat{L}_{n}\left(x_{j}\right)\right\}_{j, n=0,1, \ldots, N}$ in a stable way. Although $\hat{L}_{n}(x)=$ $L_{n}(x) e^{-x / 2}$ are well behaved for all $n$ and $x$, direct computation of $L_{n}(x)$ will produce overflows for $n$ and $x$ large. To overcome this difficulty, Funaro [8] has introduced the scaled Laguerre functions which provide a stable discrete transform. However, the scaled Laguerre functions in [8] are not suitable for the Galerkin formulation used here. Hence a new procedure to evaluate $\hat{L}_{n}(x)$ in a stable way is needed.

For any given $x$, we write

$$
\hat{L}_{n}(x)=\operatorname{sign}\left(L_{n}(x)\right)\left(d_{n}(x)\right)^{n} \text { with } d_{n}(x)=\left|L_{n}(x)\right|^{\frac{1}{n}} e^{-\frac{x}{2 n}} .
$$

We derive from (2.1) that $\operatorname{sign}\left(L_{n}(x)\right)$ and $d_{n}(x)$ can be determined from the following recurrence relations:

$$
\operatorname{sign}\left(L_{0}(x)\right)=1, \operatorname{sign}\left(L_{1}(x)\right)=\operatorname{sign}(1-x), d_{0}(x)=1, d_{1}(x)=|1-x| .
$$

Then, for $n \geq 2$,

$$
\begin{gathered}
\operatorname{sign}\left(L_{n}(x)\right)=\operatorname{sign}\left\{\frac{2 n-1-x}{n} \operatorname{sign}\left(L_{n-1}(x)\right) d_{n-1}(x)\left[\frac{d_{n-1}(x)}{d_{n-2}(x)}\right]^{n-2}\right. \\
\left.-\frac{n-1}{n} \operatorname{sign}\left(L_{n-2}(x)\right)\right\} ; \\
d_{n}(x)=\left(d_{n-2}(x)\right)^{\frac{n-2}{n}} \mid \frac{2 n-1-x}{n} \operatorname{sign}\left(L_{n-1}(x)\right) d_{n-1}(x)\left[\frac{d_{n-1}(x)}{d_{n-2}(x)}\right]^{n-2} \\
-\left.\frac{n-1}{n} \operatorname{sign}\left(L_{n-2}(x)\right)\right|^{\frac{1}{n}} .
\end{gathered}
$$

Of course when $d_{n-2}(x)=0$, the above formulae should be replaced by (see (2.1))

$$
\begin{aligned}
\operatorname{sign}\left(L_{n}(x)\right) & =\operatorname{sign}\left\{\frac{2 n-1-x}{n} L_{n-1}(x)\right\} ; \\
d_{n}(x) & =\left|\frac{2 n-1-x}{n}\right|^{\frac{1}{n}}\left(d_{n-1}(x)\right)^{\frac{n-1}{n}} .
\end{aligned}
$$

Thus $\hat{L}_{n}(x)$ can be computed in a stable way for all $n$ and $x$ by using the above recurrence relations.

\section{REFERENCES}

[1] R. Adams, Sobolev Spaces, Academic Press, New York, 1975.

[2] C. Bernardi and Y. Maday, Spectral method, in Handbook of Numerical Analysis, Vol. 5, P. G. Ciarlet and J. L. Lions, eds., North-Holland, Amsterdam, 1997, pp. 209-485. 
[3] J. P. BoyD, Orthogonal rational functions on a semi-infinite interval, J. Comput. Phys., 70 (1987), pp. 63-88.

[4] B. L. Buzbee, G. H. Golub, and C. W. Nielson, On direct methods for solving Poisson's equations, SIAM J. Numer. Anal., 7 (1970), pp. 627-656.

[5] C. Canuto, M. Y. Hussaini, A. Quarteroni, and T. A. Zang, Spectral Methods in Fluid Dynamics, Springer-Verlag, New York, 1987.

[6] O. Coulaud, D. Funaro, and O. Kavian, Laguerre spectral approximation of elliptic problems in exterior domains, Comput. Methods Appl. Mech. Engrg., 80 (1990), pp. 451-458.

[7] P. J. Davis And P. Rabinowitz, Methods of Numerical Integration, Academic Press, 1984.

[8] D. Funaro, Computational aspects of pseudospectral Laguerre approximations, Appl. Numer. Math., 6 (1990), pp. 447-457.

[9] D. Funaro, Polynomial Approximations of Differential Equations, Springer-Verlag, Berlin, 1992.

[10] D. Gottlieb and S. A. Orszag, Numerical Analysis of Spectral Methods: Theory and Applications, CBMS Regional Conf. Ser. in Appl. Math. 26, SIAM, Philadelphia, 1977.

[11] C. E. Grosch AND S. A. Orszag, Numerical solution of problems in unbounded regions: Coordinates transforms, J. Comput. Phys., 25 (1977), pp. 273-296.

[12] B. Guo, Jacobi spectral approximation and its applications to differential equations on the half line, J. Comput. Math., 18 (2000), pp. 95-112.

[13] B. Guo AND J. SHen, Laguerre-Galerkin method for nonlinear partial differential equations on a semi-infinite interval, Numer. Math., to appear.

[14] V. IRANZO AND A. FAlQuÉs, Some spectral approximations for differential equations in unbounded domains, Comput. Methods Appl. Mech. Engrg., 98 (1992), pp. 105-126.

[15] Y. Maday, B. Pernaud-Thomas, and H. Vandeven, Reappraisal of Laguerre type spectral methods, Rech. Aerospat., 6 (1985), pp. 13-35.

[16] C. MavriPlis, Laguerre polynomails for infinite-domain spectral elements, J. Comput. Phys., 80 (1989), pp. $480-488$.

[17] S. A. Orszag, Spectral methods for complex geometries, J. Comput. Phys., 37 (1980), pp. 70 92 .

[18] J. SHEn, Efficient spectral-Galerkin method I. Direct solvers of second- and fourth-order equations by using Legendre polynomials, SIAM J. Sci. Comput., 15 (1994), pp. 1489-1505.

[19] J. Shen, Efficient spectral-Galerkin method II. Direct solvers of second- and fourth-order equations by using Chebyshev polynomials, SIAM J. Sci. Comput., 16 (1995), pp. 74-87.

[20] J. SHEn, Efficient spectral-Galerkin methods III. Polar and cylindrical geometries, SIAM J. Sci. Comput., 18 (1997), pp. 1583-1604.

[21] J. SHen, Efficient spectral-Galerkin methods IV. Spherical geometries, SIAM J. Sci. Comput., 20 (1999), pp. 1438-1455.

[22] G. Szegö, Orthogonal Polynomials, 4th ed., Amer. Math. Soc. Colloq. Publ. 23, AMS, Providence, RI, 1975.

[23] T. TAng, The Hermite spectral method for Gaussian-type functions, SIAM J. Sci. Comput., 14 (1993), pp. 594-606. 